\title{
Evaluation of Visual Evoked Potential in Psoriasis Vulgaris Patients in a Tertiary Care Centre
}

\author{
T. N. Vijayalakshmi ${ }^{1}$, K. Vishnu Priya ${ }^{2}$, D. Celine $^{3}$, K. Subha Revathi ${ }^{4}$, \\ N. Saravanan ${ }^{5}$, G. Balaji6 \\ ${ }^{1}$ Associate Professor, ${ }^{2}$ IIIrd Year Post-Graduate, ${ }^{3}$ Professor \& Head, ${ }^{4}$ Senior Assistant Professor, \\ Department of Physiology, Chengalpattu Medical College, Tamil Nadu. \\ ${ }^{5}$ Professor, Department of Dermatology, Venereology \& Leprosy, Thanjavur Medical College, \\ ${ }^{6}$ Professor \& Head, Department of Ophthalmology, Chengalpattu Medical College, Chengalpattu, Tamil Nadu, \\ India. \\ Corresponding Author: K. Vishnu Priya
}

\begin{abstract}
Psoriasis is an immunologically mediated, inflammatory disease with increased risk of painful and destructive arthritis, cardiovascular morbidity and psychosocial challenges. Psoriasis vulgaris is the most common form of psoriasis, seen in approximately $90 \%$ of patients. Besides skin, other regions widely involved are bone, cardiovascular system and eye. Psoriatic eye manifestations involve many of the structures of eye; from eyelid till optic nerve. Recording of Visual evoked potential helps in studying the integrity of optic nerve and its abnormality signifies demyelination. The concept Brain skin axis proposed in literature shows involvement of nervous system with immune mediated inflammatory pathway in Psoriasis. During intense inflammatory course, highly dense sensory nerves in plaques trigger the release of neuropeptides, targeting keratinocytes and further nerve damage. With this background, the present study was aimed to assess Optic pathway integrity by Visual Evoked Potential in patients of Psoriasis vulgaris.
\end{abstract}

Materials and Methods: A Case control study was done with Institutional Ethics Committee approval and written informed consent on 100 plaque type psoriatic cases and 50 apparently healthy subjects as controls. Cases were divided into 2 groups based on their disease duration as I - new cases (1 to 5 years), II - old cases (6 to 10 years). Psoriatic patients treated with UVB and Methotrexate and with relapse of both gender of 15 to 35 years were included in the study.
Patients with Diabetes Mellitus, Hypertension, any other chronic illness and autoimmune disease, severe forms of psoriasis, patients on oral steroids, and with $\mathrm{H} / \mathrm{O}$ ocular diseases, eye surgery and neurological illness were excluded from the study. The mean Psoriasis Area Severity Index (PASI) was 16.09 \pm 11.11. According to PASI score, the cases were categorised as <10 - mild; >11-20 - moderate; and $>21$ - severe. Clinical and ophthalmological examination was done for study participants. After proper instructions to patients for VEP procedure, VEP was recorded using Physiopac NEURO PERFECT EMG 2000 SYSTEM. P100 latency of controls and cases was taken for analysis and analysed using SPSS 21.0 version.

Results: There was increase in P100 latency of cases when compared to controls. P100 latency progressively increases as the severity of disease increases.

Conclusion: Increased latency of P100 is a sign for demyelination. As this is seen in the present study, in psoriatic patients, there is a probability of optic nerve involvement during active state of disease.

Key words: Psoriasis, VEP, P100, PASI

\section{INTRODUCTION}

Psoriasis is a chronic, inflammatory condition with proliferative lesions in skin. The most characteristic lesions are red, scaly, sharply demarcated, indurated plaques, present particularly over the extensor surfaces of limbs and scalp. The 
extent and severity varies enormously over time and between individuals with common morphological variants. Both genetic and environmental influences have a critical role in the aetiology and pathogenesis of the disease. $^{[1]}$ In India, the prevalence of psoriasis in adults varies from 0.44 to $2.8 \%$. The onset of psoriasis is bimodal with two peaks - the first between 16 and $22 \mathrm{yrs}$ and the second between 57 and 60 years of age with male preponderance ${ }^{[2]}$. $\mathrm{T}$ helper cells are the key pathogenic factor in Psoriasis. Activated T1 and T17 cells secrete proinflammatory mediators including interferon (IFN)- $\gamma$, tumour necrosis factor (TNF)- $\alpha$, IL-22, and IL-17. These cytokines act in a synergistic manner to amplify keratinocyte responses in psoriatic plaques ${ }^{[3]}$. Though psoriasis was considered as purely a skin disease due to imbalance in immune system, Plaque psoriasis is now being recognized as a multi systemic disease. This is because of its associated comorbidities such as psoriatic arthritis, cardiovascular disease, metabolic syndrome, inflammatory bowel disease and depression ${ }^{[4]}$.

Further, involvement of eyes in psoriasis has been quoted in literature by several authors. Involvement of cornea, conjunctiva, eye lids, lacrimal glands, uvea and lens were reported ${ }^{[5-8]}$. Uveitis was the most reported complication in these patients [9]. The underlying pathogenesis of eye involvement is multifactorial. The integrity of visual pathway beyond retina has not been evaluated widely. Since, optic nerve function can be evaluated by VEP, the present study was attempted to assess VEP to study the involvement of optic nerve damage in psoriatic patients.

VEP is a relatively large, positive polarity wave generated in the visual cortex in response to a visual stimulus ${ }^{[10]}$. Latency is a measurement of the time in milliseconds from the onset of the stimulus presentation to the peak of the appropriate waveform component. The latency of the positive wave at a midline occipital EEG electrode is usually at 100 milliseconds after stimulation and so called the P100 ${ }^{[11]}$. $\mathrm{N}$ denotes the negative waves and $\mathrm{P}$ denotes the positive waves. The P100 peak is usually easy to recognize, highly reproducible and measurable than other two negative waves such as N75 and N145. N75 reflects the activity of fovea and primary visual cortex (area 17). P100 results from activation of primary visual cortex and also due to discharge from thalamo cortical fibres. N145 reflects the activity of visual association areas 18 and 19.

The clinical interpretation of pattern VEPs depends mainly on the P100. The normal values for P100 latency in $\mathrm{ms}$ is 96.9 \pm 3.6 (Misra and Kalita) and102.3 \pm 5.1 (Shahrokhi et al). Over repeated trials, even months and years apart, VEPs are usually very precise for an individual. P100 latency in the range of 114 to $117 \mathrm{~ms}$ is considered abnormal $^{[11]}$. An excessive difference in the latencies on the two sides (usually 6-10 ms) is also considered abnormal. P100 peak time is affected by parameters such as pattern size, pattern contrast, patient age, gender and visual acuity.

In Poland and Italy, integrity of optic nerve in psoriasis was portrayed by conducting Visual evoked potential studies. ${ }^{[12,13]}$ and pointed that optic nerve is damaged in psoriatic patients. There is existence of Brain skin axis which is mediated by mast cells, NGF, Substance - P and numerous neuropeptides. ${ }^{[14]}$.This shows that psoriasis has an immune mediated, inflammatory pathway involvement in nervous system too. A question arises whether optic nerve is targeted in psoriatic patients, with Brain skin axis as a link. Hence, the present study was conducted to explore the hidden concept of demyelination in psoriatic patients with a non-invasive tool. For this, VEP was selected and the P100 component was evaluated in controls and cases.

\section{METHODOLOGY}

This was a case control study done in Chengalpattu Medical College. Informed written consent was obtained from study participants after getting approval from 

centre.

Institutional ethics Committee. Rules of modified Helsinki's declaration were followed. 50 age and gender matched apparently healthy subjects - patient's attenders and hospital workers were chosen. 100 psoriatic patients were chosen from Outpatient department of Dermatology. They were divided into Group I - 50 new cases ( 1 to 5 years) duration, Group II - 50 old cases (6 to 10 years) duration. The mean PASI score was $16.09 \pm 11.11$. Severity was calculated with thickness, scaling and erythema and the area involved was measured with one palm surface, taken as $1 \%$ and PASI was calculated. Psoriatic patients treated with UVB and Methotrexate and with relapse of both gender of 15 to 35 years were included in the study. Patients with Diabetes Mellitus, Hypertension, any other chronic illness and autoimmune disease, severe forms of psoriasis, patients on oral steroids, and with $\mathrm{H} / \mathrm{O}$ ocular diseases, eye surgery and neurological illness were excluded from the study. Detailed history, anthropometric measurements and clinical examination was done. Ophthalmological examination-Visual acuity with E chart, pupillary light reflex, Colour vision with Ishihara's chart, and fundoscopy with Heinz ophthalmoscope after dilatation with $0.8 \%$ Tropicamide drops were carried out. The study participants were asked to come for VEP recording after hair wash. Wearing of spectacles was allowed during procedure. Pattern reversal VEP was recorded using Physiopac- NEUROPERFECT EMG 2000 SYSTEM. The study participants were made to sit $100 \mathrm{~cm}$ away from the checker board stimulus. One eye was tested at a time and the other eye was covered with hand in order to avoid stimulation of other eye. Electrodes were fixed as per 10-20 international system. Active electrode $(\mathrm{Oz})$ was placed $3 \mathrm{~cm}$ above inion, Ground electrode $(\mathrm{Cz})$ at forehead and Reference electrode $(\mathrm{Fz}) 12 \mathrm{~cm}$ above nasion. The electrodes were connected to preamplifier and electrode impedance was kept below 5 $\mathrm{k} \Omega$.Amplification range was 20,000 $1,00,000$, Low cut filter at $2 \mathrm{~Hz}$ and high cut filter at $100 \mathrm{~Hz}$ were the settings done. Sweep speed was $50 \mathrm{~ms}$, Sweep duration was $20 \mathrm{~ms} / \mathrm{D}$ and Sweep sensitivity was 1 $\mu \mathrm{V}$. 200 epochs were given for each eye. Black and white checkerboard of $80 \%$ contrast was used with pattern reversal as type of stimulus. $8 \times 8$ min was the pattern size and the rate of stimuli was1-2 Hz. Full field stimuli was used with black and white colours. The focus was red with the mean luminance of the central field $50 \mathrm{~cd} / \mathrm{m} 2$ and the background luminance of $20-40 \mathrm{~cd} / \mathrm{m} 2$. P100 peak in right and left eyes were taken for analysis. Parameters were analysed using SPSS 21.0 version.

\section{RESULTS}

Data was analysed with Descriptive statistics, Chi square test and ANOVA using SPSS software -21.0 version. $P$ value $<0.05$ is taken as statistically significant.

Table No: 1 - The baseline characteristics of Controls and Cases

\begin{tabular}{|c|c|c|c|c|}
\hline Baseline characteristics & Group & Mean & SD & Sig. \\
\hline \multirow{3}{*}{ 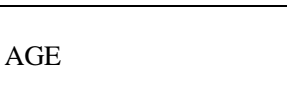 } & Control & 27.94 & 5.36 & \multirow{3}{*}{0.965} \\
\hline & New Cases & 27.92 & 4.47 & \\
\hline & Old Cases & 28.16 & 5.00 & \\
\hline \multirow{3}{*}{ HEIGHT } & Control & 155.94 & 3.33 & \multirow{3}{*}{0.471} \\
\hline & New Cases & 155.88 & 3.69 & \\
\hline & Old Cases & 155.12 & 4.08 & \\
\hline \multirow{3}{*}{ WEIGHT } & Control & 62.92 & 8.24 & \multirow{3}{*}{0.813} \\
\hline & New Cases & 62.90 & 7.20 & \\
\hline & Old Cases & 60.62 & 7.42 & \\
\hline \multirow{3}{*}{ BMI } & Control & 25.85 & 3.29 & \multirow{3}{*}{0.792} \\
\hline & New Cases & 25.91 & 3.19 & \\
\hline & Old Cases & 25.22 & 3.17 & \\
\hline
\end{tabular}

Table No: 2 -P100 latency in Right Eye between controls and cases

\begin{tabular}{|l|l|l|l|l|}
\hline Group & Mean P100 Latency & SD & F & Sig. \\
\hline Controls & 99.92 & 2.576 & & \multirow{2}{*}{7.461} \\
\cline { 1 - 2 } New Cases & 100.6 & 6.528 & \multirow{2}{*}{0.001} \\
\cline { 1 - 3 } Old Cases & 104.29 & 7.887 & & \\
\hline
\end{tabular}

Table No: 3 -P100 latency in Left Eye between controls and cases

\begin{tabular}{|l|l|l|l|l|}
\hline Group & Mean P100 Latency & SD & F & Sig. \\
\hline Controls & 100.25 & 2.929 & & \multirow{2}{*}{2.807} \\
\cline { 1 - 3 } New Cases & 100.54 & 6.364 & \\
\cline { 1 - 3 } Old Cases & 102.81 & 7.482 & & \\
\hline
\end{tabular}

Table No: 4 - Severity of disease based on Mean PASI score

\begin{tabular}{|l|l|l|}
\hline PASI & MEAN & SD \\
\hline Mild & 8.30 & 1.38 \\
\hline Moderate & 14.35 & 2.90 \\
\hline Severe & 33.22 & 10.43 \\
\hline
\end{tabular}


T. N. Vijayalakshmi et.al. Evaluation of visual evoked potential in psoriasis vulgaris patients in a tertiary care centre.

Table No: 5 - P100 Latency right and left eye among mild, moderate and severe cases based on mean PASI score

\begin{tabular}{|l|l|l|l|l|l|}
\hline P100 latency & Mild MEAN \pm SD & Moderate MEAN \pm SD & Severe MEAN \pm SD & F & P value \\
\hline Right eye & $99.64 \pm 5.26$ & $103.06 \pm 7.36$ & $106.75 \pm 8.50$ & 8.02 & 0.0005 \\
\hline Left eye & $99.16 \pm 4.32$ & $102.67 \pm 7.23$ & $104.88 \pm 8.67$ & 6.09 & 0.003 \\
\hline
\end{tabular}

\section{DISCUSSION}

Table - 1 shows statistically no significant difference in age, height, weight and BMI between controls and cases, hence the study group and controls were comparable. Table -2 and 3 depicts that the mean P100 latency in cases was higher when compared to controls. The increase in mean P100 latency in right eye of cases was highly statistically significant $(\mathrm{P}$ value 0.001). When left eye was compared, though there was increase in P100 latency, it was not statistically significant. Though there is increase in P100 latency in cases compared to controls, the differences were less than the average values of $\pm 3 \mathrm{SD}$ (Tables 2 and 3). Similarly, Grzybowski et al in Poland, observed increase in P100 latency while recording pattern reversal VEP, but it was not statistically significant. While recording pattern flash VEP, there was statistically significant increase in P100 latency in cases. Similar study was done by Perossini et al in Italy. They obtained increase in latency in $53 \%$ of cases. But, they also didn't show statistically significant increase in P100 latency in cases. These two studies concluded that optic nerve is involved in Psoriasis ${ }^{[12,13]}$.

Prolonged P100 latency implies a defect in conduction in visual pathway proximal to the optic chiasma on that side, commonly disease of optic nerve [11]. Grzybowski et al obtained normal wave pattern in only 4 cases. Similarly, Perossini et al observed VEP alterations in more than $3 / 4^{\text {th }}$ cases. Further, they obtained bifid $\mathrm{P}$ wave in 4 cases. In the present study, $20 \%$ of cases had abnormal P100 latency and 2\% had P100 latency in upper limit of normal. In new cases, 6 abnormal and 1 in upper limit of normal and in old cases, 14 abnormal and 1in upper limit of normal has been noted. The possible mechanism of optic nerve involvement in cases with abnormal range is the inflammatory process mediated by TNF- $\alpha$, interferon- $\gamma$, IL- 1,2 , 17 and 22 along with neuropeptides such as Nerve growth factor, Calcitonin Gene Related peptide and Substance P.

Ansel et al pointed out a number of non-neuronal- cells such as T cells, B cells and monocytes secretes neuropeptides. These neuropeptides are capable of activating and turning leukocytes into effector cells which release wide range of cytokines ${ }^{[15]}$. CGRP causes vasodilation, mast cell activation. NGF causes proliferation of cutaneous neurons, hyper innervation, recruitment of mast cells and $\mathrm{T}$ Cells to site of lesions. Substance $P$ causes chemotaxis of neutrophils, keratinocyte proliferation, dermal mast cell degranulation and T-Cell activation. Vasoactive Intestinal Peptide causes neurogenic inflammation, keratinocyte proliferation, formation and growth of blood vessels ${ }^{[14]}$. During active stage of disease, these cytokines and neuropeptides would have triggered optic nerve too, which could be the possible reason for increase in P100 latency in cases in the present study. Further, common embryological origin of skin and optic nerve [13] and Brain skin axis - a pathway mediated by mast cells, NGF, Substance $-\mathrm{P}$ and numerous neuropeptides ${ }^{[14]}$ could also be the possible reasons.

Zhu et al portrayed that following denervation injuries, there was remission in psoriasis. They highlighted that sensory nerve infiltration is generally higher in psoriatic plaques than normal skin ${ }^{[16]}$. This shows that neuropeptides are involved in active plaques. There exists a communication between keratinocytes, innate immune cells and nerve cells both in central and peripheral nervous system. Hence, optic nerve involvement in these patients could be neuro inflammation orchestrated by cytokine web similar to sensory nerve infiltration within psoriatic plaques. 
Table -2 and 3 shows mean P100 latency of old cases was higher than mean P100 latency of new cases. This result could be attributed to the remission and exacerbation pattern of the disease. In old cases (duration 6 to 10 years), relapse and exacerbations are fairly common and chance for entrapment of optic nerve is more. Hence, the probability of injury to myelin sheath is increased.

In the present study, $43 \%$ were mild, $34 \%$ were moderate and $23 \%$ were severe cases. Table -5 shows progressive increase in P100 latency in right and left eyes from mild to severe cases and it was highly statistically significant. Generally, increased density of neuro fibrils, Schwann cells and neural support cells are present in psoriatic plaques ${ }^{[17]}$. Biopsies of the damaged skin from psoriatic patients showed intensive expression of neuropeptides such as Substance P, CGRP and vascular intestinal peptide (VIP) in the nervous fibres. In contrast, in healthy skin, no such activity was encountered ${ }^{[12]}$. Hence, during intense inflammatory process, cytokines and neuropeptides released could possibly act as local mediators and cause epidermal proliferation as well as damage to neuro fibrils, axons and myelin sheath.

Skin, nervous system and immune system work in unison and is being studied with animal models extensively ${ }^{[17]}$. Further, fMRI studies conducted in psoriatic patients revealed that the processing of facial expressions of disgust was significantly impaired. Blood flow in the anterior insular cortex was reduced ${ }^{[18]}$. This shows that psoriasis has a hidden pathophysiology in central nervous system.

The results of the present study signify that ongoing inflammation in active lesions in psoriatic patients could trigger the optic nerve, setting in damage such as injury to myelin sheath, loss of neuro fibrils and alteration in membrane potential. This proves that there is a possibility of optic nerve being affected in Psoriasis vulgaris. The results of present study could not be compared in Indian patients due to lack of data in available literature. The underlying genetic trigger and neuro inflammatory pathway involved are yet to be identified.

\section{CONCLUSION}

Statistically significant increase in mean P100 latency of cases was obtained when compared to controls; $20 \%$ of cases had abnormal P100 latency in the present study. This denotes inflammatory pathway mediated damage to optic nerve myelin sheath would have occurred in psoriatic patients. As there is a probability of involvement of optic nerve in psoriasis vulgaris, the present study concludes that VEP might be used routinely in ophthalmic evaluation of psoriasis vulgaris patients.

\section{Limitations Of The Study:}

Cytokine estimation if done would have supported the obtained results. Head size (technical limitation of VEP) and ocular dominance of study participants, if taken into account could have added further information regarding the study.

\section{Implications:}

In screening for ophthalmic complications in psoriatic patients, VEP can also be added.

\section{Future Scope Of The Study}

Electrophysiological recordings along with molecular level studies in a larger sample can bring out this possible association with acceptable evidence.

\section{ACKNOWLEDGEMENT}

Financial Support: Fund received from ICMR, New Delhi by applying in financial assistance to $\mathrm{MD} / \mathrm{MS} / \mathrm{MCH} / \mathrm{MDS}$ Thesis/Dissertation in 2018 December slot.

Conflict of Interest: None

Source of Funding: None

Ethical Approval: Approved 


\section{REFERENCES}

1. Rooks Textbook of Dermatology,9th edition.

2. Dogra S, Mahajan R. Psoriasis: Epidemiology, clinical features, comorbidities, and clinical scoring. Indian Dermatol Online J [Internet]. 2016 [cited 2020 Jul 29];7(6):471. Available from:

http://www.idoj.in/text.asp?2016/7/6/47 1/193906

3. Fitzpatrick's Dermatology 9th edition.

4. Armstrong AW et. al. New Treatment Paradigms in Psoriasis: Understanding and Incorporating Recent and Emerging Trends. Common and Not-So-Common Comorbidities of Psoriasis. Sem Cutan Med Surg [Internet]. 2018 Feb 1 [cited 2019 Nov 29];37(2S):S48-51. Available from:

https://www.globalacademycme.com/cm e/dermatology-skin-disease-educationfoundation/new-treatment-paradigmspsoriasis-understanding/common-andnot-so-common-comorbidities-psoriasis

5. Campanati A, Neri P, Giuliodori K, Arapi I, Carbonari G, Borioni E, et al. Psoriasis beyond the skin surface: a pilot study on the ocular involvement. Int Ophthalmol [Internet]. 2015 Jun [cited 2019 Nov 29];35(3):331-40. Available from:

http://link.springer.com/10.1007/s10792 -014-9950-8

6. Chowdhury B, Pavaiya A, Khurana VK. Ocular Findings in Psoriasis Patients. 2017;4(10):4.

7. Shainhouse T. Ocular Manifestations of Psoriasis. 2017;5.

8. Algarni A, Almuqrin A, Alarwan A. Association between psoriasis and ocular disorders: A narrative review. Clin Med Invest [Internet]. 2017 [cited 2020 Aug 6];2(2). Available from: http://oatext.com/Association-betweenpsoriasis-and-ocular-disorders-Anarrative-review.php

9. Au S, Yaniv S, Gottlieb AB. Psoriatic Eye Manifestations. Psoriasis Forum [Internet]. 2011 Sep [cited 2019 Nov 29];17a(3):169-79. Available from: http://journals.sagepub.com/doi/ 10.1177/247553031117a00301

10. Jasper R.Daube,Devon I.Rubin Clinical Neurophysiology 3rd edition.

11. Visual evoked potentials - Frank W.Drislane. In.

12. Grzybowski A, Grzybowski G, Druzdz A, Zaba R. Visual evoked potentials in patients with psoriasis vulgaris. :8.

13. Perossini M, Turio E, Perossini T, Romagnoli M, Benedetti S, Cei G, et al. Pattern VEP Alterations in Psoriatic Patients may Indicate a Sub Clinic Optic Neuritis. Doc Ophthalmol [Internet]. 2005 Mar [cited 2019 Nov 29];110(23):203-7. Available from: http://link.springer.com/10.1007/s10633 -005-4830-1

14. Paus R, Theoharides TC, Arck PC. Neuroimmunoendocrine circuitry of the 'brain-skin connection.' Trends in Immunology [Internet]. 2006 Jan [cited 2020 Oct 21];27(1):32-9. Available from:

https://linkinghub.elsevier.com/retrieve/ pii/S1471490605002644

15. Ansel JC, Armstrong CA, Song I, Quinlan KL, Olerud JE, Caughman SW, et al. Interactions of the Skin and Nervous System. Journal of Investigative Dermatology Symposium Proceedings [Internet]. 1997 Jan [cited 2020 Nov 29];2(1):23-6. Available from:

https://linkinghub.elsevier.com/retrieve/ pii/S1087002415301854

16. Zhu TH, Nakamura M, Farahnik B, Abrouk M, Lee K, Singh R, et al. The Role of the Nervous System in the Pathophysiology of Psoriasis: A Review of Cases of Psoriasis Remission or Improvement Following Denervation Injury. Am J Clin Dermatol [Internet]. 2016 Jun [cited 2020 Oct 18];17(3):257-63. Available from: http://link.springer.com/10.1007/s40257 -016-0183-7

17. Ayasse MT, Buddenkotte J, Alam M, Steinhoff M. Role of neuroimmune 
T. N. Vijayalakshmi et.al. Evaluation of visual evoked potential in psoriasis vulgaris patients in a tertiary care centre.

circuits and pruritus in psoriasis. Exp Dermatol [Internet]. 2020 Apr [cited 2020 Nov 29];29(4):414-26. Available from:

https://onlinelibrary.wiley.com/doi/abs/1 $0.1111 /$ exd.14071

18. Zangeneh FZ, Shooshtary FS. Psoriasis Types, Causes and Medication. In: Lima $\mathrm{H}$, editor. Psoriasis - Types, Causes and Medication [Internet]. InTech; 2013 [cited 2020 Aug 6]. Available from: http://www.intechopen.com/books/psori asis-types-causes-and-

medication/psoriasis-types-causes-andmedication

How to cite this article: T. N. Vijayalakshmi, K. Vishnu Priya, D. Celine et.al. Evaluation of visual evoked potential in psoriasis vulgaris patients in a tertiary care centre. International Journal of Research and Review. 2021; 8(6): 148-154. DOI:https://doi.org/10.52403/ijrr.20210618 